\title{
INTERVENÇÃO PRECOCE EM ESCOLARES DE RISCO PARA A DISLEXIA: REVISÃO DA LITERATURA
}

\author{
Early intervention in students at risk for dyslexia: literature review
}

\author{
Maíra Anelli Martins ${ }^{(1)}$, Simone Aparecida Capellini ${ }^{(2)}$
}

\begin{abstract}
RESUMO
Tema: intervenção precoce em escolares de risco para a dislexia. Objetivo: este estudo tem por objetivo geral mapear os artigos publicados sobre intervenção com escolares de risco para dislexia e, como objetivos específicos, analisar descritivamente aspectos específicos dos textos. Conclusão: as publicações na área em relação ao tema não são constantes, porém, os artigos científicos analisados evidenciam a preocupação dos pesquisadores em elaborar, desenvolver e validar instrumentos de avaliações e intervenções que contribuam para a identificação precoce da dislexia.
\end{abstract}

DESCRITORES: Reabilitação; Dislexia; Leitura; Aprendizagem

\section{INTRODUÇÃO}

A dislexia é caracterizada como um distúrbio específico de aprendizagem de origem neurológica, em que o escolar encontra dificuldade com a fluência correta na leitura e dificuldade na habilidade de decodificação e soletração, resultante de um déficit em componentes da linguagem ${ }^{1}$.

Ao longo dos anos a dislexia vem sendo estudada sob a perspectiva do diagnóstico e da intervenção ${ }^{2}$. Estudos têm demonstrado que escolares em situação de risco para a dislexia apresentam falha de processamentos auditivo e visual e que em decorrência dessas falhas o acionamento de mecanismos cognitivos para analisar, sintetizar, manipular, estocar e evocar informações linguísticas encontra-se alterado, prejudicando, assim, a aprendizagem do princípio alfabético de sistemas de escrita ${ }^{3-5}$

(1) Aluna do Curso de Fonoaudiologia da Faculdade de Filosofia e Ciências da Universidade Estadual Paulista - FFC/ UNESP, Marília,SP, Brasil; Bolsista de Apoio Técnico CNPq.

(2) Fonoaudióloga; Docente do Departamento de Fonoaudiologia e Programa de Pós-Graduação em Educação da Faculdade de Filosofia e Ciências da Universidade Estadual Paulista - FFC/UNESP, Marília, SP,Brasil; Doutora e Pós-Doutora em Ciências Médicas pela Faculdade de Ciências Médicas da Universidade Estadual de Campinas, FCM/UNICAMP, Campinas, SP.

Conflito de interesses: inexistente
Por isso, há aproximadamente 30 anos, estudos vêm investigando os resultados da aplicação de programas de intervenção com habilidades cognitivo-linguísticas, com o objetivo de minimizar o impacto das falhas de processamento auditivo, visual e fonológico em escolares que apresentam dificuldades de aprendizagem ou dislexia ${ }^{6-9}$

Com a finalidade de iniciar cada vez mais precocemente a identificação da dislexia, estudos apontam para a necessidade de se realizar intervenção precoce nos escolares em fase inicial de alfabetização - pré-escola e $1^{\circ}$ e $2^{\circ}$ anos escolares - para que os fatores preditivos para o bom desempenho em leitura, como: conhecimento do alfabeto, nomeação automática rápida, repetição de nãopalavras e habilidades de consciência fonológica, sejam trabalhados nos escolares que apresentam desempenho abaixo do esperado em relação ao seu grupo-classe, escolares estes denominados na literatura internacional como de risco para a dislexia ${ }^{10-14}$

Os primeiros programas de intervenção com crianças de risco para a dislexia descritos na literatura datam da década de $80^{15-17}$ Pesquisadores ${ }^{18-22}$ desenvolveram programas de remediação para 0 tratamento das dificuldades de leitura por meio do treinamento da consciência fonológica e ensino explícito das regras de correspondência grafofonêmicas. Esses programas são geralmente baseados em atividades fonêmicas, silábicas e suprafonêmicas (rima e aliteração), que têm por objetivo 
desenvolver habilidades referentes ao processamento fonológico relacionado à leitura e compreensão textual|23-26

Autores mostraram que escolares de risco para a dislexia quando submetidos a programas de intervenção apresentam melhoras significantes em habilidades cognitivo-linguísticas, como na habilidade de percepção dos sons, identificação de letra, de sílabas e de palavras e medidas mais avançadas das habilidades de alfabetização, como, por exemplo, fluência na leitura, vocabulário e compreensão de leitura, diminuindo o fracasso escolar e o número de escolares considerados maus leitores e de risco ${ }^{27-29}$

No Brasil ainda são escassas as pesquisas com enfoque na identificação precoce da dislexia e consequentemente na intervenção precoce com estes escolares. Na literatura nacional| ${ }^{30-35}$, os estudos enfocam o desenvolvimento de programas com base fonológica e leitura com escolares já diagnosticados com dislexia ou distúrbio de aprendizagem.

Com base no exposto, este estudo tem por objetivo geral mapear os artigos publicados sobre intervenção com escolares de risco para a dislexia e, como objetivo específico, analisar descritivamente os aspectos específicos dos textos: caracterização geral do texto, aspectos específicos de caracterização do estudo, caracterização da pesquisa e especificação dos dados.

\section{MÉTODO}

Este estudo caracteriza-se por uma revisão de literatura sistemática, assim como outros tipos de estudo de revisão, trata-se de uma forma de pesquisa que utiliza como fonte de dados a literatura sobre determinado tema ${ }^{36}$ A base de dados escoIhida para a pesquisa de artigos com informações a respeito de métodos de intervenção analisados constitui-se em bancos de dados internacionais ERIC, ScienceDirect, Pubmed, Lilacs, disponíveis em serviços online.

A pesquisa na base de dados iniciou-se com a busca dos seguintes descritores em língua inglesa, combinando dois ou três termos: kindergarteners, reading intervention; at-risk readers; reading instruction; learning disabilities; response to intervention; early intervention. E, em língua portuguesa: programas de intervenção precoce, leitores de risco, intervenção precoce. O período para coleta das informações foi do primeiro semestre de 2005 ao primeiro semestre de 2009.

Os resumos obtidos no banco de dados foram comparados entre si para verificação de superposição de artigos. Posteriormente, os resumos foram analisados para selecionar, ainda preliminarmente, os trabalhos que atendessem aos critérios de inclusão, ou seja, os trabalhos deveriam considerar instrumentos de intervenção apenas com crianças de risco e a faixa etária dos sujeitos dos estudos deveria estar entre 4 e 8 anos de idade.

Nesta fase de coleta de dados, foram selecionados $30(85,71 \%)$ resumos, os quais foram numerados sequencialmente, e uma nova etapa de busca dos artigos completos permitiu chegar à definição final dos textos que efetivamente foram analisados neste estudo, em um total de 17 (48,57\%) artigos.

Os artigos completos encontrados foram numerados de acordo com os resumos e uma nova análise foi realizada segundo os critérios de exclusão, isto é, os artigos científicos não deveriam ser de intervenção com escolares que apresentassem comorbidade, apenas deveriam apresentar programas de intervenção com escolares de risco para a dislexia. Além disso, foram excluídos os resumos que não disponibilizaram os artigos científicos na íntegra. Assim, este estudo foi composto por 17 artigos científicos, sendo $16(94,57 \%)$ internacionais e 1 $(5,88 \%)$ nacional.

Os dados coletados foram analisados por meio de registro em ficha, que continha os seguintes itens: a) caracterização geral do texto: autores, ano, financiamento para a realização da pesquisa e local de pesquisa; b) aspectos específicos de caracterização do estudo: instrumentos utilizados para intervenção de escolares de risco, número e gênero dos participantes, caracterização da amostra (idade e proveniência), caracterização dos sujeitos como grupo único ou experimental/controle; c) caracterização da pesquisa: tipo de pesquisa, tipo de intervenção utilizada e profissional responsável e; d) especificação dos dados: finalidade do uso do instrumento de intervenção, validação e sujeitos como meio de intervenção. As informações coletadas nas fichas de registro foram codificadas numericamente e distribuídas em planilhas de Excel. Os resultados foram analisados e estão descritos em duas tabelas e uma figura.

\section{REVISÃO DA LITERATURA}

A análise dos resultados considerou 17 artigos, encontrados na base de dados ERIC, ScienceDirect, Pubmed, Lilacs, no período do segundo semestre de 2005 ao segundo semestre de 2009, cujo tema refere-se à intervenções precoces em escolares de risco para a dislexia.

A figura 1 apresenta a distribuição dos artigos de acordo com o ano de publicação, sendo possível observar um maior número de publicações nos anos de 2005 e 2008. Conforme descrito na literatura ${ }^{37-42}$, 


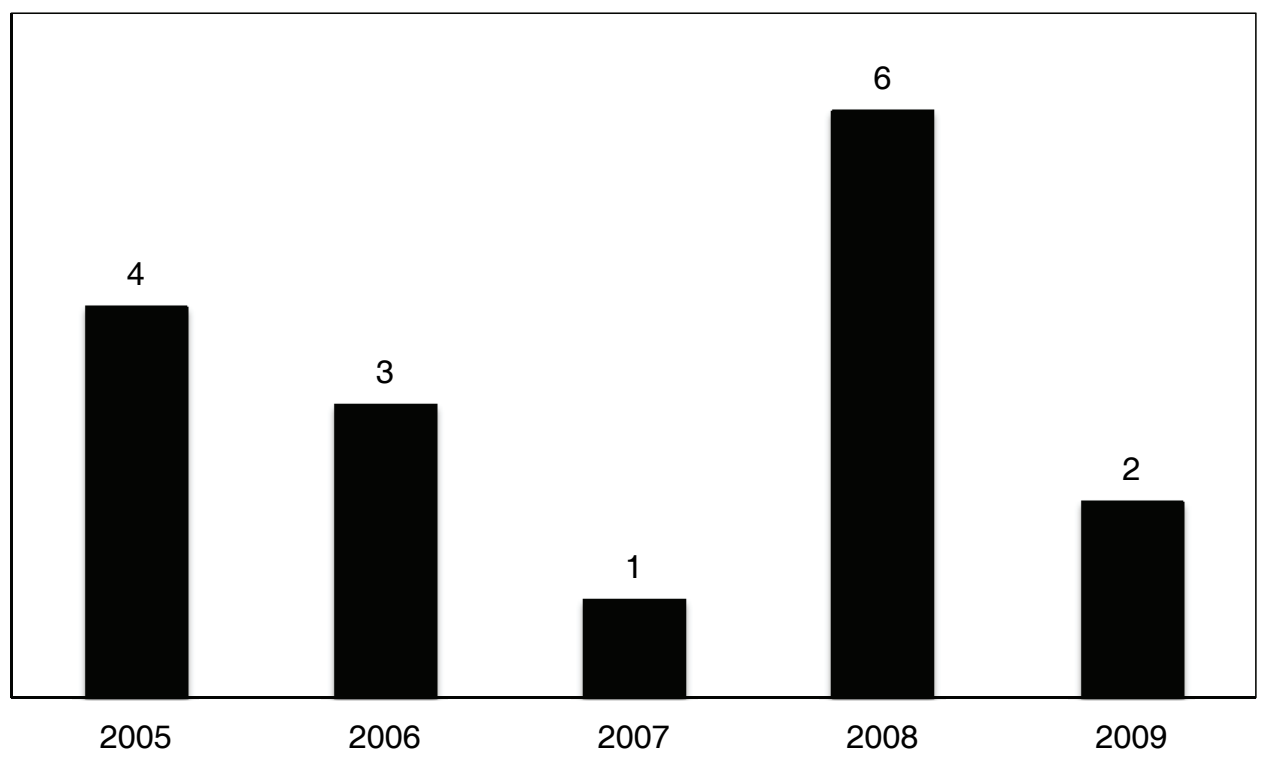

Figura 1 - Distribuição da frequência dos artigos científicos publicados entre 2005 e 2009

ocorreu aumento no número de publicações em 2008 em comparação aos demais anos, corroborando os achados deste estudo, que revelou 6 artigos científicos $(35,29 \%)$ publicados sobre a temática programas de intervenção com escolares de risco para a dislexia.

Dos 17 artigos pesquisados, 14 (82,35\%) são artigos publicados nos Estados Unidos - EUA, $2(11,76 \%)$ publicados na Europa, sendo um na Holanda e outro no Reino Unido e 1 (5,88\%) artigo publicado no Brasil. Destes estudos, apenas 2 $(11,76 \%)$ artigos não foram financiados por agências governamentais de auxílio à pesquisa ${ }^{40,41}$.

Com base nesses achados, pode-se considerar que ainda são poucas as pesquisas desenvolvidas com escolares de risco para a dislexia, com a finalidade de identificação e diagnóstico precoce desta condição de origem genético-neurológica. O país que apresenta maior número de artigos publicados é os Estados Unidos, que apresenta como critério diagnóstico para a dislexia a falta de resposta à intervenção ${ }^{14,43-45}$.

A escassa produção científica com esta temática gera um desconhecimento sobre o perfil do escolar de risco para a dislexia, fazendo com que todos os escolares que apresentem falhas no processo de alfabetização sejam considerados eletivos para o diagnóstico de dislexia, isto associado ao fato de que no Brasil, a alfabetização não é baseada no ensino do princípio alfabético da Língua Portuguesa, o que gera, muitas vezes, diagnósticos incorretos de dislexia ou transtorno de aprendizagem, conforme descrito na literatura nacional ${ }^{46}$.
A maior parte dos artigos, ou seja, 10 (58,82\%) dos 17 artigos selecionados para a composição deste estudo aplicaram apenas 1 programa de intervenção em escolares de risco para dislexia e $7(41,18 \%)$ artigos utilizaram um conjunto de 2 ou mais programas de intervenção para verificar a eficácia da ação terapêutica empregada. Isto mostra que ainda não há um consenso na literatura sobre qual a forma de intervenção mais eficaz para identificar precocemente a dislexia em escolares em fase inicial de alfabetização, conforme descrito na literatura internacional ${ }^{47-49}$.

Entretanto, o mesmo não ocorre quando a temática se refere à identificação dos sinais de risco para a dislexia em escolares em fase inicial de alfabetização. A literatura internacional ${ }^{50-52}$ destaca os principais fatores de risco como sendo: fala ininteligível; imaturidade fonológica; redução de léxico; dificuldade em aprender o nome das letras ou os sons do alfabeto; dificuldade para entender instruções, compreender a fala ou material lido; dificuldade para lembrar números, letras em sequência, questões e direções; dificuldade para lembrar sentenças ou estórias; atraso de fala; confusão direita-esquerda, embaixo, em cima, frente-atrás (palavras-conceitos) e dificuldade em processar os sons das palavras.

Com base nos sinais de risco para a dislexia, pesquisadores identificaram cinco componentes essenciais necessários para o sucesso da aprendizagem da leitura em crianças da pré-escola: a consciência fonológica, conhecimento dos fonemas, fluência, vocabulário e compreensão, sendo este o caminho para uma leitura fluente e para a compreensão de textos ${ }^{41}$. 
Assim, os 17 estudos (100\%) analisados utilizaram testes de consciência fonológica para a identificação precoce de escolares com sinais da dislexia e como base para a elaboração de estratégias dos programas de intervenção, a rima e a aliteração. Além disso, também foram utilizadas estratégias de nomeação automática rápida, vocabulário, correspondência letra-som e ortografia, aspectos necessários para a aprendizagem da leitura e da escrita em um sistema de escrita com base alfabética.

Os testes citados nos artigos científicos e utilizados para mensurar o fator "risco" foram: Woodcock-Johnson Psychoeducational Test Battertudosy, Dynamic Indicators of Basic Early Literacy Skills (DIBELS) e Peabody Picture Vocabulary Test. No Brasil, o procedimento utilizado foi o Teste para a identificação precoce dos problemas de leitura ${ }^{27,46}$

A literatura ${ }^{53-55}$ descreve que o risco para a dislexia deve ser identificado na faixa etária de 5 a 8 anos de idade. Apesar de neste estudo os artigos científicos apresentarem variabilidade no número de escolares que compuseram as amostras dos estudos (tabela 1), foi possível verificar que 16 (94,08\%) das intervenções foram realizadas com escolares em fase inicial de alfabetização (tabela 2). Isto porque o objetivo de se realizar intervenção com escolares de risco para a dislexia é

Tabela 1 - Distribuição da frequência dos participantes nos artigos científicos

\begin{tabular}{ccc}
\hline Participantes & freq. & perc. \\
\hline menos de 50 & 3 & $17,65 \%$ \\
50 a 100 & 4 & $23,53 \%$ \\
101 a 200 & 4 & $23,53 \%$ \\
201 a 300 & 2 & $11,76 \%$ \\
301 a 400 & 1 & $5,88 \%$ \\
401 a 500 & 1 & $5,88 \%$ \\
mais de 500 & 1 & $5,88 \%$ \\
sem especificação & 1 & $5,88 \%$ \\
Total & 17 & $100,00 \%$ \\
\hline
\end{tabular}

Tabela 2 - Frequência dos participantes quanto a faixa etária nos artigos científicos

\begin{tabular}{ccc}
\hline Faixa etária & freq. & perc. \\
\hline 5 a 8 anos & 16 & $94,08 \%$ \\
$\pm 4,09$ anos & 1 & $5,88 \%$ \\
Total & 17 & $100,00 \%$ \\
\hline
\end{tabular}

verificar se, após a realização de programas específicos com as habilidades cognitivo-linguísticas necessárias para a alfabetização e aprendizagem do princípio alfabético, os escolares com sinais da dislexia apresentam melhora na aprendizagem da leitura ou se permanecem com as habilidades cognitivo-linguísticas alteradas, o que significaria que estes escolares apresentam realmente uma defasagem e que, por isso, deveriam ser submetidos a avaliações interdisciplinares para a investigação da existência do quadro de dislexia, uma vez que a falta de resposta à intervenção pode ser considerada um critério para este diagnóstico $0^{6,10,33}$

Neste estudo, em 16 artigos (94,08\%), os escolares foram provenientes de escolas e em 1 artigo $(5,88 \%)$ os escolares foram provenientes de escolas pré-primárias privadas e locais de cuidados infantis. Quanto ao delineamento metodológico dos estudos, foram verificadas as intervenções com grupos experimentais e controle em 12 artigos (70,59\%) e, em 5 artigos $(29,41 \%)$ somente com grupos experimentais, o que aponta para a tendência de uso de grupo controle, com o objetivo de controlar as variáveis acadêmicas e sociais durante a realização da intervenção e, assim, melhor detalhar e verificar a eficácia dos programas de intervenção, conforme descrito na literatura internacional $22,26-28,41,42,45,47-49,54,55$

Nos artigos científicos analisados, foi identificado que as intervenções foram realizadas com escolares, pais e professores. Em 13 artigos (76,47\%) foi utilizada a intervenção apenas com escolares, e em 3 artigos (17,65\%) foi utilizada a intervenção com escolares e professores e em apenas 1 artigo $(5,88 \%)$ foi utilizada a intervenção com escolares, professores e pais.

Este dado é significante, pois é possível observar que a recomendação do "Center for the Improvement of Early Reading Achievement" não está sendo seguida para trabalhos com enfoque na melhoria da leitura. Este Centro recomenda 10 princípios básicos para o desenvolvimento da leitura em séries iniciais de alfabetização, sendo um destes princípios o desenvolvimento de programas de intervenção precoce com a leitura que incluem a participação da família e dos professores ${ }^{56-58}$

Apenas 3 (17,65\%\%) artigos deste estudo informaram que as intervenções foram realizadas por fonoaudiólogos, sendo que em 10 (58,82\%) as intervenções foram realizadas pelo professor no contexto da sala de aula. Este dado evidencia o quanto é importante a participação do professor tanto na detecção como na intervenção precoce com os sinais da dislexia, partindo-se do pressuposto que este professor está em contato constante com estes escolares e que a sua atuação é fundamental para a melhoria da leitura e a verificação do 
escolar que apresenta alguma alteração cognitivolinguística que compromete o seu desempenho em leitura e compreensão da leitura $22,27,42,46$

\section{CONCLUSÃO}

A partir dos resultados encontrados neste estudo, pode-se concluir que as publicações internacionais sobre o uso de programas de intervenção com escolares que apresentam risco para dislexia vêm tomando considerável importância nos últimos anos. Todavia, os números de publicações brasileiras são extremamente inferiores aos das publicações internacionais, evidenciando a necessidade da continuidade de pesquisas que desenvolvam ou adaptem programas de intervenção utilizados internacionalmente para a realidade brasileira.

Posteriormente à realização do mapeamento dos artigos, pode-se concluir que as publicações na área em relação ao tema não são constantes, porém os artigos científicos analisados evidenciam a preocupação dos pesquisadores em elaborar, desenvolver e validar instrumentos de avaliações e intervenções que contribuam para a identificação precoce da dislexia.

\section{AGRADECIMENTOS}

Ao CNPq pela concessão da bolsa de apoio técnico à primeira autora e da bolsa de produtividade em pesquisa à segunda autora.

\begin{abstract}
Background: early intervention in students at risk for dyslexia. Purpose: this study aims to map the general articles on intervention with students at risk for dyslexia and specific objectives, descriptively analyzing specific text aspects. Conclusion: there are few published data on this issue; however, the reviewed scientific articles highlight the concern of the researchers to elaborate, develop and validate assessments and interventions that contribute to the early identification of dyslexia.
\end{abstract}

KEYWORDS: Rehabilitation; Dyslexia; Reading; Learning

\section{REFERÊNCIAS}

1. Lyon GR, Shaywitz SE, Shaywitz BA. Defining dyslexia, comorbidity, teachers' knowledge of language and reading a definition of dyslexia. Ann Dyslexia. 2003;53:1-14.

2. Aaron PG, Joshi RM, Gooden R, Bentum KE. Diagnosis and Treatment of Reading Disabilities Based on the Component Model of Reading. J Learn Disabil. 2008;41(1):67-84.

3. Ziolkowski RA, Goldstein H. Effects of an Embedded Phonological Awareness Intervention During Repeated Book Reading on Preschool Children With Language Delays. J of Early Intervention. 2008;31(1):67-90.

4. Betourne LS, Patti SF. Phonological processing and oral language abilities in fourth-grade poor readers. J Commun Disord. 2003;36(6):507-27.

5. Capovilla AGS, Capovilla FC. Efeitos do treino de consciência fonológica em crianças com baixo nível sócio-econômico. Psicol. Reflex. Crit. 2000;13(1):7-24.
6. Bradley L, Bryant PE. Categorising sounds and learning to read a causal connection. Nature. 1983;301:419-21.

7. Broom YM, Doctor EA. Developmental phonological dyslexia: a case study of the efficacy of a remediation programme. Cognit. Neuropsychol. 1995;12(7):725-66.

8. Torgensen JK, Davis C. Individual difference variables that predict response to training in phonological awareness. J. Exp. Child. Psychol. 1996;63:1-21.

9. Gonzalez OMR, Espinel AIG, Rosquete RG. Remedial interventions for children with reading disabilities: Speech perception effective component in phonological training? J. Learn. Disab. 2002;35(4):334-42.

10. Hamilton SS, Glascoe FP. Evaluation of children with reading difficulties. Am Fam Physician. 2006;74(12):2079-84.

11. Scanlon DM, Vellutino FR, Small SG, Fanuele DP, Sweeney JM. Severe Reading Difficulties-Can They Be Prevented? A Comparison of Prevention and Intervention Approaches. Exceptionality. 2005;13(4):209-27. 
12. Schneider W, Roth E, Ennemoser M. Training phonological skills and letter knowledge in children at risk for dyslexia: a comparison of three kindergarten intervention programs. J. Educ. Psy. 2000;92(2):284-95.

13. Petursdottir AL, McMaster K, McComas JJ, Bradfield $\mathrm{T}$, Braganza $\mathrm{V}$, Koch-McDonald $\mathrm{J}$ et al. Brief experimental analysis of early reading interventions. J Sch Psychol. 2009;47:215-43.

14. Gijsel MAR, Bosman AMT, Verhoeven L. Kindergarten risk factors, cognitive factors, and teacher judgments as predictors of early reading in dutch. J. Learn Disab. 2006;39(6):558-71.

15. Graminha SSV, Machado VLS, Francischini EL, Befi VM. Emprego de um procedimento de treino gradual de discriminação de sílabas em crianças com dificuldades na leitura e escrita. Arq. Bras. Psicol. 1987;39(1):84-94.

16. Bebetto AMBF. Remediação de leitura e escrita em escolares através de instrução programada. [Dissertação]. São Paulo (SP): Instituto de Psicologia, Universidade de São Paulo; 1981.

17. Braga SML. Remediação da leitura: um estudo com escolares de primeiro grau utilizando a técnica de cloze. [Dissertação]. São Paulo (SP): Instituto de Psicologia, Universidade de São Paulo; 1981.

18. Calhoon MB. Effects of a peer-mediated phonological skill and reading comprehension program on reading skill acquisition for middle school students with reading disabilities. J. Learn. Disab. 2005;38(5):424-33.

19. Agnew JA, Dorn C, Eden GF. Effect of intensive training on auditory processing and reading skills. Brain Lang. 2004;88:21-5.

20. Ygual-Fernández A, Cervera-Mérida JF. Valoración del riesgo de dificultades de aprendizaje de la lectura en niños con trastornos del lenguaje. Rev. Neurol. 2001;2:95-106.

21. Tressoldi PE, Vio C, lozzino R. Efficacy of an intervention to improve fluency in children with development dyslexia in a regular ortography. J. Learn. Disab. 2006;40(3):203-9.

22. O'Connor RE, Fulmer D, Harty KR, Bell KM. Layers of reading intervention in kindergarten through third grade: changes in teaching and student outcomes. J Learn Disab. 2005;38(5):440-55.

23. Phillips BM, Clancy-Menchetti J, Lonigan CJ. Successful Phonological Awareness Instruction With Preschool Children: Lessons From the Classroom. Top Early Child Spec Educ. 2008;28(1):3-17.

24. Koutsoftas AD, Harmon MT, Gray S. The Effect of Tier 2 Intervention for Phonemic Awareness in a Response-to-Intervention Model in Low-Income Preschool Classrooms. Lang Speech Hear Serv Sch. 2009;40:116-30.
25. Wolf M, Barzillai M, Gottwald S, Miller L, Spencer $\mathrm{K}$, Norton $\mathrm{E}$ et al. The RAVE-O Intervention: Connecting Neuroscience to the Classroom. Mind Brain Educ. 2009;3(2):84-93.

26. Bailet LL, Repper KK, Piasta SB, Murphy SP. Emergent Literacy Intervention for Prekindergarteners at Risk for Reading Failure. J Learn Disabil. 2009;42(4):336-55.

27. Cardoso RKOA, Capellini SA. Eficácia do programa de intervenção com a consciência fonológica em escolares com risco para a dislexia. Rev. psicopedag. 2009;26(81):367-75.

28. Gunn B, Smolkowski K, Biglan A, Black C, Blair J. Fostering the Development of Reading Skill through Supplemental Instruction: Results for Hispanic and Non-Hispanic Students. J Spec Educ. 2005;39(2):66-85.

29. Ryder JF, Tunmer WE, Greaney KT. Explicit instruction in phonemic awareness and phonemically based decoding skills as an intervention strategy for truggling readers in whole language classrooms. Read Writ. 2007;21(4):349-69.

30. Salgado CA, Capellini SA. Programa de remediação fonológica em escolares com dislexia do desenvolvimento. Pró-Fono R. Atual. Cient. 2008;20(1):31-6.

31. Capellini SA, Padula NAMR, Ciasca SM. Desempenho de escolares com distúrbio específico de leitura em programa de remediação. Pró-Fono R. Atual Cient. 2004;16(3):261-74.

32. Germano GD, Capellini SA. Eficácia do programa de remediação auditivo-visual computadorizado em escolares com dislexia. Pró-Fono R. Atual. Cient. 2008; 20(4):237-42.

33. Silva CD, Capellini SA. Eficácia do programa de remediação fonológica e leitura no distúrbio de aprendizagem. Pró-Fono R. Atual. Cient. 2010;22(2):131-8.

34. Pinheiro FH, Capellini SA. Treinamento auditivo em escolares com distúrbio de aprendizagem. Pró-Fono R. Atual. Cient. 2010;22(1):49-54.

35. Capellini SA, Sampaio MN, Kawata KHS, Padula NAMR, Santos LCA, Lorencetti MD et al. Eficácia terapêutica do programa de remediação fonológica em escolares com dislexia do desenvolvimento. Rev. CEFAC. 2010;12(1):27-39.

36. Sampaio RF, Mancini MC. Systematic review studies: a guide for careful synthesis of the scientific evidence. Rev. bras. fisioter. 2007;11(1):83-9.

37. Simmons DC, Coyne MD, Kwok O, McDonagh S, Harn BA, Kame'enui EJ. Indexing Response to Intervention: A Longitudinal Study of Reading Risk From Kindergarten Through Third Grade. J Learn Disabil. 2008;41(2):158-73.

38. Kamps D, Abbott M, Greenwood C, Wills $H$, Veerkamp M, Kaufman J. Effects of Small-Group 
Reading Instruction and Curriculum Differences for Students Most at Risk in Kindergarten: Two-Year Results for Secondary- and Tertiary-Level Interventions. J Learn Disabil. 2008;41(2):101-14. 39. Bowyer-Crane C, Snowling MJ, Duff FJ, Fieldsend E, Carroll JM, Miles $\mathrm{J}$ et al. Improving early language and literacy skills: differential effects of an oral language versus a phonology with reading intervention. J Child Psychol Psychiatry. 2008;49(4):422-32.

40. Harn BA, Linan-Thompson S, Roberts G. Intensifying Instruction: Does Additional Instructional Time Make a Difference for the Most At-Risk First Graders? J Learn Disabil. 2008;41(2):115-25.

41. Wanzek J, Vaughn S. Response to Varying Amounts of Time in Reading Intervention for Students With Low Response to Intervention. J Learn Disabil. 2008;41(2):126-42.

42. Scanlon DM, Gelzheiser LM, Vellutino FR, Schatschneider S, Sweeney JM. Reducing the Incidence of Early Reading Difficulties: Professional Development for Classroom Teachers vs. Direct Interventions for Children. Learn Individ Differ. 2008;18(3):346-59.

43. Fletcher JM, Vaughn S. Response to Intervention: Preventing and Remediating Academic Difficulties. Child Dev Perspect. 2009;3(1):30-7.

44. Torgesen JK. The Response to Intervention Instructional Model: Some Outcomes From a LargeScale Implementation in Reading First Schools. Child Dev Perspect. 2009;3(1):38-40.

45. Linan-Thompson S, Vaughn S, Prater K, Cirino PT. The Response to Intervention of English Language Learners at Risk for Reading Problems. J Learn Disabil.2006;39(5):390-8.

46. Capellini SA, Sampaio MN, Fukuda MTM, Oliveira AM, Fadini CC, Martins MA. Protocolo de identificação precoce dos problemas de leitura: estudo preliminar com escolares de $1^{\circ}$ ano escolar. Rev. psicopedag. 2009;26(81):367-75.

47. O'Connor RE, Harty KR, Fulmer D. Tiers of Intervention in Kindergarten Through Third Grade. J Learn Disabil. 2005;38(6):532-8.

http://dx.doi.org/10.1590/S1516-18462011005000028

RECEBIDO EM: 22/09/2010

ACEITO EM: 16/11/2010

Endereço para correspondência:

Simone Aparecida Capellini

Avenida Hygino Muzzy Filho, 737

Marília - SP

CEP: $17-525-900$

E-mail: sacap@uol.com.br
48. Vellutino FR, Scanlon DM, Small S, Fanuele DP. Response to Intervention as a Vehicle for Distinguishing Between Children With and Without Reading Disabilities: Evidence for the Role of Kindergarten and First-Grade Interventions. J Learn Disabil. 2006;39(2):157-69.

49. Smit-Glaudé SWD, Strien JWV, Licht R, Bakker DJ. Neuropsychological Intervention in Kindergarten Children with Subtyped Risks of Reading Retardation. Ann Dyslexia. 2005;55(2):217-45.

50. ASHA: American Speech and Hearing Association. Language-based learning disabilities. [cited 2009 Aug 20]. Available from: http://www. asha.org.

51. Etchepareborda MC. La intevencíón en los transtornos disléxicos: entrenamiento de la conciencia fonológica. Rev Neurol. 2003;36(1):13-9. 52. Capellini SA. Distúrbios de aprendizagem versus dislexia. In: Ferreira LP, Befi-Lopes DM, Limongi SCO, Eds. Tratado de fonoaudiologia. São Paulo: Roca; 2004. p. 862-76.

53. Simmons DC, Kame'enui EJ, Harn B, Coyne MD, Stoolmiller M, Santoro LE et al. Attributes of Effective and Efficient Kindergarten Reading Intervention: An Examination of Instructional Time and Design Specificity. J Learn Disabil. 2007;40(4):331-47.

54. Vaughn S, Linan-Thompson S, Mathes PG, Cirino PT, Carlson CD, Pollard-Durodola SD et al. Effectiveness of Spanish Intervention for First-Grade English Language Learners at Risk for Reading Difficulties. J Learn Disabil. 2006;39(1):56-73.

55. Lo $\mathrm{Y}$, Wang $\mathrm{C}$, Haskell $\mathrm{S}$. Examining the Impacts of Early Reading Intervention on the Growth Rates in Basic Literacy Skills of At-Risk Urban Kindergarteners. J Spec Educ. 2009;43(1):12-28.

56. CIERA: Center for the Improvement of Early Reading Achievement. Improving the reading achievement of America's children: 10 researchbased principles. [cited 2010 Aug 29]. Available from: http://www.ciera.org/library/instresrc/principles/ index.html

57. Fielding-Barnsley R, Purdie N. Developing Pre-Literacy Skills via Shared Book Reading: Assessment of a family intervention program for pre-school children at risk of becoming reading disabled. Aust J Learn Disabil. 2002;7(3):13-9.

58. Fielding-Barnsley R, Purdie N. Early intervention in the home for children at risk of reading failure. Support for Learning: British Journal of Learning Support. 2003; 18(2):77-82. 